\title{
Maxillo-facial radiology case 156
}

\author{
SADJ November 2017, Vol 72 no 10473
}

CJ Nortjé

A 69-year old female, referred by her general practitioner, presented with the complaint of a burning sensation in her oral cavity. The upper jaw was edentulous. The patient herself and the oral mucosa appeared healthy and there was no obvious cause for the burning sensation. A routine pantomograph revealed marginal resorption of the alveolar crests, suggestive of an underlying periodontitis. Two years later she presented with a non-healing socket after a mobile 34 had been extracted three months previously. Figure 1 is a cropped pantomograph at the stage when she originally presented and Figure 2 was recorded when she presented for the second time. What are the important radiological features and what is your provisional diagnosis?
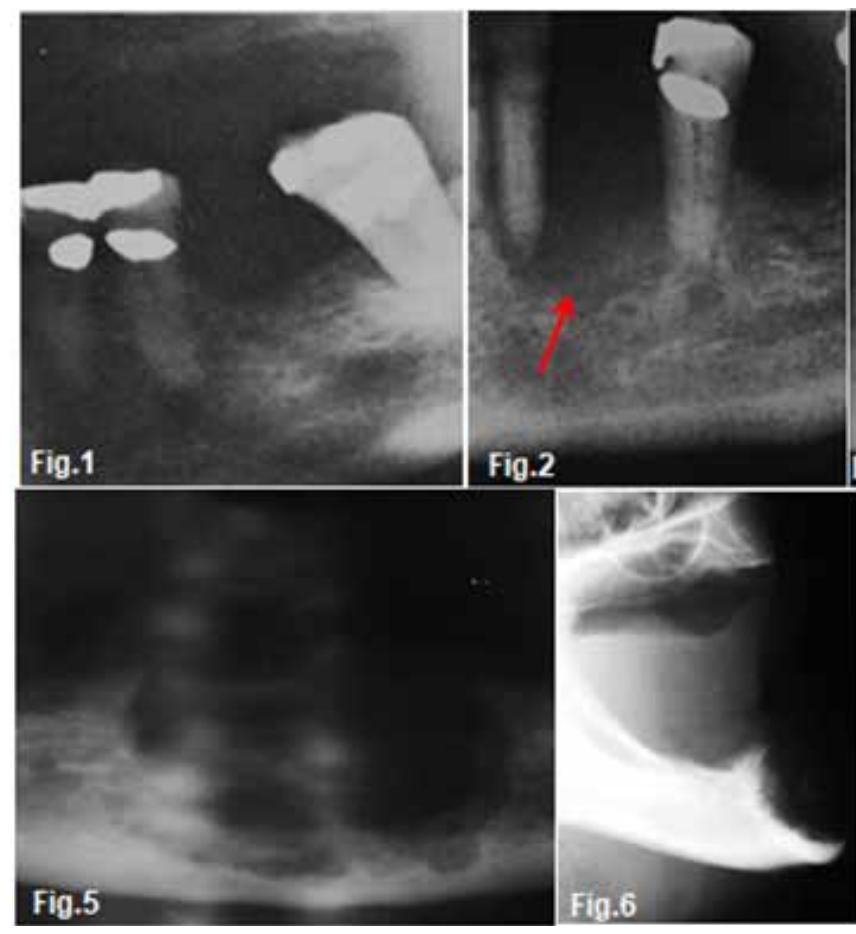
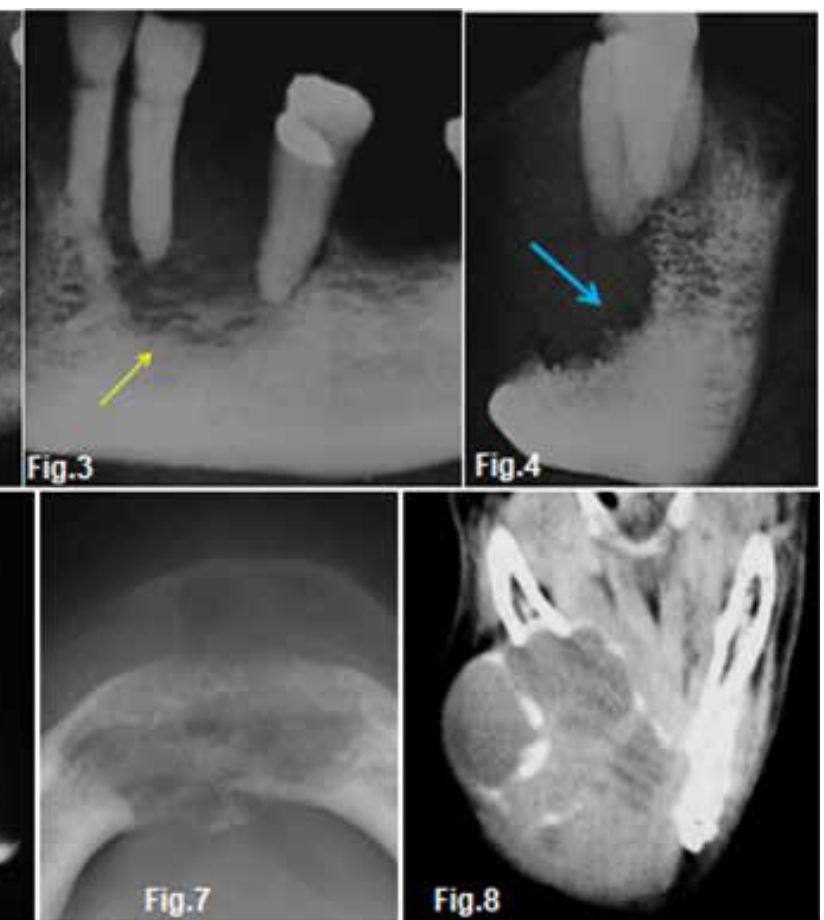

\section{INTERPRETATION}

The cropped pantomograph (Fig.1) shows marginal resorption of the alveolar crests while Figure 2 shows a nonspecific area of rarefaction in the canine-premolar region (red arrow) without evidence of sclerosis at the margins of the lesion. Figures $3 \& 4$ are radiographs of the resected specimen showing the changes more clearly. Permeative changes, lack of marginal sclerosis (yellow arrow) and absence of periosteal reaction indicate a malignant process but do not confirm a specific disease. The "geographic area" results from total destruction of the buccal half of the mandible (blue arrow). A histological examination confirmed the diagnosis of a primary intraosseous carcinoma.

Figures 5, 6\&7 are from a similar case in a 70 year old edentulous female patient, the diagnosis in her instance being a clear cell odontogenic carcinoma. Figure 5 shows radiolucencies of varying size with an irregular outline, Figure 6 shows destruction of the buccal part of the mandible while Figure 7 shows a moth-eaten appearance and lack of marginal sclerosis. Figure 8 is a CT scan of another case showing a very destructive lesion involving the body and ramus of the right mandible. Bony flecks are discernible within the lesion.

Most carcinomas found inside the jaws have originated from the squamous epithelium covering the alveolar ridge, the gingiva or the floor of the mouth. Primary intraosseous carcinoma of the jaw is a very rare tumour. It is classified by the $\mathrm{WHO}$ as an odontogenic carcinoma which is a squamous cell carcinoma arising within the jaw, having no initial contact with the oral mucosa and presumably developing from residues of the odontogenic epithelium. The tumour occurs mainly in adults in the sixth to seventh decades, male-tofemale ratio is $3: 1$, and the growth is usually situated in the posterior mandible. The lesion may be asymptomatic or painful and may mimic localized periodontal disease. Of practical importance is that mobility of the teeth or nonhealing of an extraction socket could well be the first clinical signs of odontogenic carcinoma. Paraesthesia and "floating" teeth may be evident and some teeth may have exfoliated spontaneously. One of the earliest and most characteristic signs of malignancy is the lack of a well-defined border with, or most frequently without, marginal sclerosis.

\section{Reference}

1. C.W van Wyk, A. Padayachee, CJ Nortje and U von der Heyden. Primary intraosseous carcinoma involving the anterior mandible. British Journal of Oral \& Maxillofacial Surgery, 1987; 25: 427-32. 